\title{
Post-vaccination Immunity Against Hepatitis B Virus and Predictors for Non-responders Among Medical Staff
}

\author{
Roohangiz Nashibi ${ }^{1}$; Seyed Mohammad Alavi ${ }^{1,}$; Farid Yousefi ${ }^{1}$; Shokrolah Salmanzadeh ${ }^{1}$; \\ Sassan Moogahi ${ }^{1}$; Fatemeh Ahmadi ${ }^{1}$; Mehdi Farashahinejad ${ }^{2}$ \\ ${ }^{1}$ Health Research Institute, Infectious and Tropical Diseases Research Center, Ahvaz Jundishapur University of Medical Sciences, Ahvaz, IR Iran \\ 2 Department of Infectious Diseases, Razi Hospital, Ahvaz Jundishapur University of Medical Sciences, Ahvaz, IR Iran \\ *Corresponding author: Seyed Mohammad Alavi, Health Research Institute, Infectious and Tropical Diseases Research Center, Ahvaz Jundishapur University of Medical Sciences, \\ Ahvaz, IR Iran. Tel: +98-6133387724, Fax:+98-6133335396, E-mail: alavi.seyedmohammad@yahoo.com, alavi-sm@ajuma.ac.ir \\ Received: April 20, 2014; Revised: July 27, 2014; Accepted: September 21, 2014
}

\begin{abstract}
Background: Hepatitis B virus (HBV) is a major cause of chronic hepatitis, liver cirrhosis, and hepatocellular carcinoma (HCC). HB vaccination is an essential step in the prevention of the disease and its consequent complications.

Objectives: Immune status of medical personnel in teaching hospitals of Khuzestan is not well known. Since these personnel are usually at risk of needle stick and other high-risk events, some challenges exist in infection control committee with regard to managing these events. This study was conducted to assess post-vaccination immunity status and non-response to HBV vaccine as well as its predictors among medical staff in a teaching hospital affiliated to Ahvaz Jundishapur University of Medical Sciences (AJUMS) in Ahvaz, southwest Iran.

Patients and Methods: In this retrospective cross-sectional study, the medical staff of a teaching hospital was evaluated for their immune response against HBV and factors affecting it. The study conducted in Razi Hospital, Ahvaz City, southwest of Iran, in 2013. Demographical, clinical, and laboratory data registered in medical files of hospital staff were analyzed by SPSS software version 16 using chi-square and Fisher exact tests. Differences with Pvalue $<0.05$ were considered significant. To identify predictors for non-responders, we used odds ratio (OR) with $95 \%$ confidence interval (CI).

Results: Out of 239 participants, $43(18 \%$ ) were men and 196 (82\%) were women. Their mean age was $31.9 \pm 18.1$ years (range of 20 - 55 years). Fourteen (5.9\%) participants were non-responders, 37 (15.5\%) were poor responders, and 188 (78.6\%) were good responders. The nonresponders were older $(>50$ years $)$ than the responders $(\mathrm{P}=0.0001)$, while the body mass index $(\mathrm{BMI})$ was not significantly different $(\mathrm{P}=$ 0.37) between them. Diabetes mellitus (DM) (OR: 7.3, 95\% CI, 1.3 - 41.7, $\mathrm{P}=0.05)$, and using immunosuppressive drugs (ISD) (OR: 3.2, 95\% CI, $1.1-11.5, \mathrm{P}=0.03$ ) were two variables in association with non-response to $\mathrm{HB}$ vaccine.

Conclusions:Non-response rate to HB vaccine in ourstudy was approximately6\%. Age over 50 years, DM, and receiving immunosuppressive drugs may be considered as predictors for non-response to HB vaccine in medical staff.
\end{abstract}

Keywords: Hepatitis B virus; Vaccination; Long-acting immunity; Immunology; Ahvaz

\section{Background}

More than 400 million people are infected with hepatitis $B$ virus worldwide. This virus is a major cause of chronic hepatitis, liver cirrhosis, and hepatocellular carcinoma (HCC) $(1,2)$. Approximately 1.2 million deaths occur due to $\mathrm{HBV}$ in the world per year (2). Hepatitis B vaccination is the essential step in the prevention of the disease and its complications. As post-vaccine immunity will prevent infection and its serious complications, HBV vaccine has been identified as the first vaccine to prevent cancer (3-5). Post-vaccine seroconversion against HBV in healthy children (like adults) is approximately 90\% (5-7). Factors that may be involved in the immune response to the vaccine and decrease the vaccine immunogenicity include age ( $\geq 40$ years), obesity, genetics, smoking, immune deficiency for any reason, male gender, technical errors in vaccine storage (such as freezing), non-compliance with the cold chain, and subcutaneous or intramuscular injection (3, 6-9).

Disease prevention and providing protective immunity for some occupational groups that have frequent contact with the virus are of particular importance. One of the most important groups in this regard is the medical staff at the hospitals (1-3, 6, 8, 10-13). HBV vaccination is required at the time of employment for these people (11). The vaccine is injected 3 times based on the country's immunization program i.e. expanded program of immunization (EPI). One to two months after the final dose of vaccine, serum level of anti-HBS antibody is measured. Those whose antibody level is more than $10 \mathrm{mIU} / \mathrm{mL}$, are called the responders (14). Typically, 90\% of vaccinated people develop a serum antibody level of higher than $10 \mathrm{mIU} /$

Copyright (C) 2015, Ahvaz Jundishapur University of Medical Sciences. This is an open-access article distributed under the terms of the Creative Commons Attribution-NonCommercial 4.0 International License (http://creativecommons.org/licenses/by-nc/4.0/) which permits copy and redistribute the material just in noncommercial usages, provided the original work is properly cited. 
$m L$ (responders) (6). Those who remain non-responder should receive another round of vaccination. Usually half the people who get the second round of HBV vaccine become the responder (15). Primary and booster vaccination with HBV in the first year of life induce long-lived immunological memory against hepatitis B in children who had been primed and boosted via EPI in Iran. These data are remarkably similar to data obtained from different parts of the world (1).

\section{Objectives}

Immune status of medical personnel in training hospitals of Khuzestan is not well known. Since these personnel are frequently at risk of needle stick and other high-risk activities, there are some challenges in infection control committee with regard to managing these events. This study was conducted to assess post-vaccination immunity status and to identify the non-response people to HBV vaccine as well as its predictors among medical staff in a teaching hospital.

\section{Patients and Methods}

In a cross-sectional study, which was conducted from 1 May to 31 August 2013, medical records of hospital staff were reviewed. Post-vaccination immunity against hepatitis B (1 - 6 months after the last dose of 3 vaccine dose) of medical staff in Razi Hospital was studied. Data were extracted from medical files and entered in a pre-designed questionnaire. Inclusion criteria were all staff who received three doses of the HBV vaccine, according to the EPI at the beginning of employment. Exclusion criteria were those whose immune status after vaccination was not clear. Data included the level of antibodies against hepatitis B, age, sex, weight, height, underlying disease, immunosuppressive drug use, immune deficiency status, blood and blood products transfusion, immunoglobulin received before vaccinations, and any other factors that may have effects on the immune response were entered into the questionnaire.

Body mass index (BMI) was calculated based on the formula; weight $(\mathrm{kg}) /$ height squared $\left(\mathrm{m}^{2}\right)$. The subjects with anti-HBs antibody titer less than $10 \mathrm{mIU} / \mathrm{mL}$ were considered as non-responders, those who were between 10 and $100 \mathrm{mIU} / \mathrm{mL}$ as poor responders, and those with 100 $\mathrm{mIU} / \mathrm{mL}$ or more were considered as good responder. The subjects were divided into two groups; non-responders, and responders and compared with regard to the factors mentioned above. Data were analyzed by SPSS software version 16 using chi-square and Fisher exact tests. Differences with P value $<0.05$ were considered significant. To identify predictors for non-responders, we used odds ratio (OR) with $95 \%$ confidence interval (CI).

\section{Results}

In this study, 239 medical staff who met the inclusion criteria was studied. Out of 239 participants, 43 (18\%) were men and 196 (82\%) were women. Their mean age was 31.9 \pm 18.1 years (range of 20 - 55 years). Of all studied cases, 14 (5.9\%) were non-responders, 37 (15.5\%) poor responders, and $188(78.6 \%)$ good responders. Comparing responder to non-responder groups (Table 1), there was no significant differences between men and women regarding the immune response $(\mathrm{P}=0.72)$. Furthermore, antibody titers in persons under 50 years was significantly different from people over 50 years old $(P=0.04)$. Mean age and BMI in both groups are shown in the Table 2. The non-responders were older than the responders ( $P$ $=0.0001)$. Although the difference in BMI was not statistically significant $(P=0.37)$, the non-responders had higher BMI than responders. As shown in Table 1, history of blood or blood products transfusion were evaluated for immune response; however, significant differences were not observed $(\mathrm{P}=0.61)$. Also, chronic underlying diseases showed no significant differences in the immune response $(\mathrm{P}=0.43)$.

Routine vaccination (EPI) history in childhood had no influence on the immune response $(\mathrm{P}=0.88)$. There was no significant difference $(\mathrm{P}=0.62)$ between the immune response and service providing departments (internal medicine, surgical, and intensive care units). Among chronic underlying diseases such as cardiovascular, bronchial asthma, chronic obstructive pulmonary diseases (COPD), diabetes mellitus (DM), and immunosuppressive drug (ISD) users, only DM (OR: 7.3, 95\% CI, 1.3 41.7, P = 0.05) and ISD use (OR: 3.2, 95\% CI, $1.1-11.5, \mathrm{P}=$ $0.03)$ were associated with non-response to $\mathrm{HB}$ vaccine (Table 3).

\section{Discussion}

Chronic HBV infection is the major cause of liver cirrhosis and HCC worldwide. The disease and its outcome are preventable with HBV vaccination. Vaccination of the health care workers is very important because of occupational exposure. Knowledge of the immune status of the personnel after complete vaccination is required for hospital infection control activities such as accidental needle stick injuries. In the present study, a good response was observed in $78.6 \%$ of the medical staff. In previous studies, Alimonos et al. (16) Zeeshan et al. (1) and Varshochi et al. (2) a good antibody response was reported at $92 \%, 86 \%$, and $96.6 \%$, respectively, which were significantly higher than that of our study. Platkov and colleagues reported a rate of $36.5 \%$ for good response in their study, which was significantly lower than our finding (12). Besides the economic, social, and racial differences, which explain the different responses to vaccines in various areas, differences in the subjects, type of vaccines used, and the manufacturer may also be the reason for various results. Hepatitis B vaccine preparation was faced with serious problems because of Iran unjust sanctions; directed by the USA and its Western confederates. Therefore, different vaccines (manufactured in Iran and other countries) with various qualities were 
Nashibi R et al.

used in the past few years. However, Pasteur Institute of Iran is a high quality organization, making the most of the vaccine available, but a portion of the vaccine, which was sometimes imported from other countries and used, has a low quality.

In this study, significant relationship was observed between the age and immune response, but other factors such as gender, underlying comorbidities (except for DM and ISD), BMI, history of childhood immunization, blood or blood products transfusion, and service providing department did not have any association with immune response. In our study, the variables of age ( $<50$ years) and BMI of 31 (although statistically nonsignificant in comparison with 33.4) were associated with higher amount of anti-HBs antibodies. Our finding about the effect of age and female gender on good response to $\mathrm{HBV}$ vaccine was in agreement with some previous studies, but in disagreement with some other studies $(1,2,6,8,13,17)$. Obesity in some studies was introduced as a predictor for non-response to HB vaccination $(13,16)$.

Platkov et al. in their study found no association between immune response, place of medical service providing and demographic factors (12). In Varshochi study, a significant inverse relationship between age and antibody serum level was reported, but such a relationship between immune response and such factors of height, weight, and body masses index have not been found (2). Alimonos and colleagues reported that host factors such as age $<50$ years, being female, BMI of 24 for women and 29 for men, and non-smoking are predictors of good response to the HBV vaccine (16). Zeeshan et al. found that the age $<50$ years and female gender were associated with a high immune response, but smoking has been associated with lower immune response (1). If the interval time between the last dose of vaccine and antibody measurement was longer, the amount of antibody would be less $(1,6,18)$. The medical staff that have had hepatitis $B$ vaccine through routine immunization schedule in childhood and has also received the vaccine at the time of employment in the hospital should have a high immune response $(7,10)$. Previous studies have suggested the high coverage of Hepatitis B vaccination in Iran due to implementation of HBV vaccination in routine childhood vaccination since 1993 all over the country (19-21).
Table 1. Demographic and Factors Affecting on Post-vaccine Immune Response Among Medical Staff in Razi Hospital, Ahvaz, $\operatorname{Iran}^{\mathrm{a}}$

\begin{tabular}{lccc}
\hline Factor & $\begin{array}{c}\text { Non Re- } \\
\text { sponder } \\
(\mathbf{n = 1 4})^{\mathrm{b}}\end{array}$ & $\begin{array}{c}\text { Good Re- } \\
\text { sponder } \\
(\mathbf{n}=\mathbf{2 2 5})^{\mathrm{b}}\end{array}$ & \\
\hline Gender & & \multicolumn{3}{c}{0} & 0.72 \\
\hline Male & $3(21.4)$ & $40(17.8)$ & \\
\hline Female & $11(78.6)$ & $185(82.2)$ & \\
\hline Age,y & & & 0.04 \\
\hline$>50^{c}$ & $3(21.4)$ & $12(5.3)$ & \\
\hline$<50$ & $11(78.6)$ & $213(94.7)$ & \\
\hline $\begin{array}{l}\text { Blood/blood product trans- } \\
\text { fusion }\end{array}$ & & & 0.61 \\
\hline Positive & $0(0)$ & $8(3.6)$ & \\
\hline Negative & $14(100)$ & $217(96.4)$ & \\
\hline Comorbidity & & & 0.43 \\
\hline Positive & $8(57.1)$ & $102(45.3)$ & \\
\hline Negative & $6(42.9)$ & $123(54.7)$ & \\
\hline Childhood vaccination & & & 0.88 \\
\hline Positive & $14(100)$ & $223(99.1)$ & \\
\hline Negative & $0(0)$ & $2(0.9)$ & \\
\hline Hospital Ward & & & \\
\hline Int. medicine & $3(21.4)$ & $50(22.2)$ & \\
\hline Surgery & $8(57.2)$ & $112(49.8)$ & \\
\hline ICU & $3(21.4)$ & $63(28)$ & \\
\hline A & & & \\
\hline
\end{tabular}

\footnotetext{
a Abbreviations: ICU, intensive care unit.

b Data are presented as No. (\%).

c Statistically significant.
}

Table 2. Comparison of Mean of Age and Body Mass Index of Studied Medical Staff in Razi Hospital, Ahvaz, Iran

\begin{tabular}{lccc}
\hline Variable & Non-responder $^{\mathrm{a}}$ & Responder $^{\mathrm{a}}$ & PValue \\
\hline Age, $\mathbf{y}$ & $44.6 \pm 9.2$ & $31.8 \pm 10.1$ & 0.0001 \\
$\begin{array}{l}\text { Body Mass Index, } \\
\text { Kg/m }\end{array}$ & $33.4 \pm 5.6$ & $31.6 \pm 7.5$ & 0.37 \\
\hline
\end{tabular}

${ }^{\mathrm{a}}$ Data are presented as Mean $\pm \mathrm{SD}$

Table 3. Comparison of Co-Morbidities Among Studied Medical Staff in Razi hospital, Ahvaz, Iran ${ }^{\text {a }}$

\begin{tabular}{|c|c|c|c|c|}
\hline Comorbidity & Non-responder $(n=14)^{b}$ & Responder $(\mathbf{n}=225)^{b}$ & Odds Ratio (95\% CI) & P Value \\
\hline Diabetes Mellitus $^{\mathrm{C}}$ & $2(14.3)$ & $6(2.7)$ & $7.3,1.3-41.7$ & 0.05 \\
\hline Immunosuppressive Drugs using ${ }^{\mathrm{C}}$ & $5(35.7)$ & $30(13.3)$ & $3.2,1.1-11.5$ & 0.03 \\
\hline Cardiovascular Diseases & $1(7.1)$ & $36(16.0)$ & $0.40,0.05-3.18$ & 0.70 \\
\hline COPD & 0 & $10(4.4)$ & NA & 0.54 \\
\hline Others & 0 & $20(8.9)$ & NA & 0.30 \\
\hline
\end{tabular}

\footnotetext{
a Abbreviations: CI, confidence interval; COPD, chronic obstructive pulmonary diseases.

b Data are presented as No. (\%).

C Statistically significant.
} 
Shamsizadeh and colleagues reported that over $75 \%$ of children in Ahvaz were seropositive 5 years after hepatitis $B$ vaccination. They concluded that because of immune memory, most of seronegative children are immune against hepatitis B (19). Alavi and colleagues in their study among hospitalized IDU have reported Hepatitis B prevalence as 3.6\%. He has concluded that lower hepatitis $\mathrm{B}$ prevalence, in comparison with hepatitis $\mathrm{C}$ prevalence (30.9\%), confirms the efficacy of the high coverage of HBV vaccination among IDU cases as well as general population who received this vaccine in childhood (20). Thus, good immune response in our personnel prior to age 50 and then its decline at older ages is justified and in agreement with other studies. Because approximately all of studied medical staff has been vaccinated in childhood, there was no significant difference in immune response with regard to childhood immunization.

We found no relation between female gender and good immune response to HB vaccine. In most previous studies, immune responses in females were higher than males $(1,3,6)$. In a study from Iran (Zamani et al.), no relation between sex and immune response was reported (8). Apparently, Iranian men do not affect non-response state. However, exact reason is not clear to us and further study is recommended. None of the cited studies found any association between immune response and service providing department or ward $(1,2,12,16)$. We think that the ward where health workers serve in, owing to booster effect of frequent HBV exposure can affect the immune response. However, health workers rotate in different hospital sections during their service, and the effect of the department on their immune response may be mimicked.

As expected, the immune response was low in medical staff with underlying diseases such as DM and those who have a history of ISD use. Diabetes role in reducing the immune response was documented in previous studies. In Leonardi (17) and Tkachenkov (13) study, DM has been introduced as a predictor of non-responder. Smoking is a well-known risk factor for diminished immune response after vaccination. As smoking in most Iranian hospitals is forbidden and smoking in health providers is considered as a stigma, about the information obtained about smoking from HCWs should be considered with caution. For this reason and fear of misinformation, smoking issue was not investigated in this study.

To our knowledge, a few studies regarding this topic have been conducted in our country, therefore this study can be considered as a work to fill this information gap. The study design is retrospective and confined to one hospital. Future population based studies are needed to generalize these results to the whole medical staff in the region. In sum, non-response rate to $\mathrm{HB}$ vaccine in our study was approximately $6 \%$. Age over 50 years, DM, and receiving immunosuppressive drugs (e.g. corticosteroid) may be considered as predictors for non-response to $\mathrm{HB}$ vaccine in medical staff.

\section{Acknowledgements}

We greatly appreciate the chief and staff of Medical Archive of Razi Hospital and Infectious Diseases Research Center affiliated to Ahvaz Jundishapur University of Medical Sciences for their kindly cooperation. We also acknowledge Dr Tabesh for his valuable assistance in the analysis of the data.

\section{Funding/support}

This study is supported by Jundishapur Infectious and Tropical Diseases Research Center.

\section{Authors' contributions}

Sassan Moogahi, Mehdi Farashahinejad, and Fatemeh Ahmadi: data collection and writing the manuscript; Roohangiz Nashibi, Shokrolah Salmanzadeh: data collection and writing the manuscript; Seyed Mohammad Alavi: final revision and primary design; and Farid Yousefi: primary design and data analysis.

\section{References}

1. Zeeshan M, Jabeen K, Ali AN, Ali AW, Farooqui SZ, Mehraj V, et al. Evaluation of immune response to Hepatitis B vaccine in health care workers at a tertiary care hospital in Pakistan: an observational prospective study. BMC Infect Dis. 2007;7:120.

2. Varshochi M, Mahmodian R. Immunologic Response to Hepatitis B Vaccine in Health Care Workers: A Screening Program and Evaluation of Some Host-Related Factors Role. Am J Immunol. 2011;7(1):12-6.

3. Chathuranga LS, Noordeen F, Abeykoon AM. Immune response to hepatitis B vaccine in a group of health care workers in Sri Lanka. Int J Infect Dis. 2013;17(11):e1078-9.

4. Amponsah-Dacosta E, Lebelo RL, Rakgole JN, Burnett RJ, Selabe SG, Mphahlele MJ. Evidence for a change in the epidemiology of hepatitis B virus infection after nearly two decades of universal hepatitis B vaccination in South Africa. J Med Virol. 2014;86(6):918-24.

5. Chang MH. Impact of hepatitis B vaccination on hepatitis B disease and nucleic acid testing in high-prevalence populations. $J$ Clin Virol. 2006;36 Suppl 1:S45-50.

6. Al Ghamdi SS, Fallatah HI, Fetyani DM, Al-Mughales JA, Gelaidan AT. Long-term efficacy of the hepatitis B vaccine in a high-risk group. J Med Virol. 2013;85(9):1518-22.

7. Spradling PR, Xing J, Williams R, Masunu-Faleafaga Y, Dulski T, Mahamud A, et al. Immunity to hepatitis B virus (HBV) infection two decades after implementation of universal infant HBV vaccination: association of detectable residual antibodies and response to a single HBV challenge dose. Clin Vaccine Immunol. 2013;20(4):559-61.

8. Zamani F, Fallahian F, Hashemi F, Shamsaei Z, Alavian SM. Immune response to hepatitis $B$ vaccine in health-care workers. Saudi Jidney Dis Transpl. 2011;22(1):179-84.

9. Posteraro B, Pastorino R, Di Giannantonio P, Ianuale C, Amore R, Ricciardi $\mathrm{W}$, et al. The link between genetic variation and variability in vaccine responses: systematic review and meta-analyses. Vaccine. 2014;32(15):1661-9.

10. Pati NT, Sethi A, Agrawal K, Agrawal K, Kumar GT, Sukriti., et al Low levels of awareness, vaccine coverage, and the need for boosters among health care workers in tertiary care hospitals in India. J Gastroenterol Hepatol. 2008;23(11):1710-5.

11. Maltezou HC, Poland GA. Vaccination policies for healthcare workers in Europe. Vaccine. 2014;32(38):4876-80.

12. Platkov E, Shlyakhov E, Glick Y, Khalemsky S, Fischbein A. Immunologic evaluation of hepatitis B vaccine application in hospital staff. Int J Occup Med Environ Health. 2003;16(3):249-53. 
13. Tkachenkov LI, Maleev VV, Putrenok LS. [Evaluation of seroconversion after vaccination of medical staff against HBV infection]. Zh Mikrobiol Epidemiol Immunobiol. 2013(5):69-74.

14. Salehi M, Alavian SM, Tabatabaei SV, Izadi S, Sanei Moghaddam E, Amini Kafi-Abad S, et al. Seroepidemiology of HBV infection in South-East of iran; a population based study. Iran Red Crescent Med J. 2012;14(5):283-8.

15. Su FH, Chu FY, Bai CH, Lin YS, Hsueh YM, Sung FC, et al. Efficacy of hepatitis $B$ vaccine boosters among neonatally vaccinated university freshmen in Taiwan. J Hepatol. 2013;58(4):684-9.

16. Alimonos K, Nafziger AN, Murray J, Bertino JS, Jr. Prediction of response to hepatitis B vaccine in health care workers: whose titers of antibody to hepatitis B surface antigen should be determined after a three-dose series, and what are the implications in terms of cost-effectiveness? Clin Infect Dis. 1998;26(3):566-71.

17. Leonardi S, Vitaliti G, Garozzo MT, Miraglia del Giudice M, Mar- seglia G, La Rosa M. Hepatitis B vaccination failure in children with diabetes mellitus? The debate continues. Hum Vaccin Immunother. 2012;8(4):448-52.

18. Hudu SA, Malik YA, Niazlin MT, Harmal NS, Adnan A, Alshrari AS, et al. Antibody and immune memory persistence post infant hepatitis B vaccination. Patient Prefer Adherence. 2013;7:981-6.

19. Shamsizadeh A, Makvandi M, Shoshtari G. Prevalence of anti hepatitis B surface antibody among children in Ahvaz, Iran, five years after vaccination. Jundishapur J Microbiol. 2011;4(2):49-54.

20. Alavi SM, Behdad F. Seroprevalence study of hepatitis C and Hepatitis B virus among hospitalized intravenous drug users in Ahvaz, Iran (2002-2006). Hepat Mon. 2010;10(2):101-4.

21. Bahmani MK, Khosravi A, Mobasser A, Ghezelsofla E. Seroprevalence of hepatitis B virus infection and vaccination compliance among health care workers in Fars Province, Iran. Iranian J Clin Infect Dis. 2010;5(1):45-50. 